decline. Therefore, we may conclude that while genetic susceptibility to schizophrenia conveys developmental cognitive deficit, it does not result in an ongoing cognitive decline, at least in later life. This, in turn, disproves the Kraepelinan notion of schizophrenia as a genetically determined progressively deteriorating brain disease.

\section{P07 LONG-TERM TRENDS IN LUNG CANCER INCIDENCE IN UK NON-SMOKERS: A COHORT STUDY OF 3.7 MILLION PEOPLE}

LJ Horsfall*, G Rait. Research Department of Primary Care and Population Health, UCL, London, UK

\subsection{6/jech-2020-SSMabstracts. 103}

Background There have been reports that lung cancer in nonsmokers (LCINS) is increasing in the UK but it is unclear whether this simply reflects fewer people smoking cigarettes or changing environmental risk factors such as increased emissions from domestic combustion. We examined UK-wide sociodemographic trends in the incidence of LCINS.

Methods We identified a cohort of 3,679,831 people selfreporting to their general practice physician as non-smokers from The Health Improvement Network (THIN) IQVIA ${ }^{\text {TM }}$ Medical Research Data. Using multivariable Poisson regression, we estimated gender-specific time-trends in the incidence of LCINS for 1998-2018 and explored the impact of geographic location, social deprivation and urban-rural classification.

Results The analysis included 3,121 cancer events and 16,051,244 person-years (PYs). Earlier time periods, high social deprivation, urban living, and residing in the North of England were associated with higher age-adjusted LCINS rates in men. Living in the North of England was the only clear risk factor for women. Between 1998-2008, age-adjusted rates in men declined by $9 \%$ per year (95\%CI: 7-11\%) from an estimated 5.6 to 1.5 per 10,000 PYs and then remained stable. These time trends for men were similar across sociodemographic variables. Between 1998 and 2007, incidence rates were stable for women at 1.3 per 10,000 PYs. However, for the least socially deprived rates increased by around 5\% per year (95\%CI: 2-9\%) from an estimated 1.3 per 10,000 PYs in 2008 to 2.1 in 2018.

Conclusion The incidence of LCINS has reduced or remained stable for most of the UK with the exception of women living in the least socially deprived areas.

\section{P08 THE PSYCHOSOCIAL DETERMINANTS OF QUALITY OF LIFE IN BREAST CANCER SURVIVORS: A SCOPING REVIEW}

${ }^{1} \mathrm{MG}$ Culbertson, ${ }^{1} \mathrm{~KB}$ Bennett, ${ }^{2} \mathrm{CK}$ Kelly, ${ }^{3} \mathrm{LS}$ Sharp, ${ }^{1} \mathrm{CC}$ Cahir* ${ }^{1}$ Division of Population Health Sciences, Royal College of Surgeons in Ireland, Dublin 2, Ireland; ${ }^{2}$ Mater Misericordiae University Hospital, Dublin 1, Ireland and Cancer Trials, Ireland; ${ }^{3}$ Population Health Sciences Institute, Newcastle University Centre for Cancer, Newcastle University, Newcastle, UK

\subsection{6/jech-2020-SSMabstracts. 104}

Background Breast cancer care today involves state-of-the-art biomedical treatment but can fail to address the broader psychosocial and quality-of-life (QoL) issues associated with the transition to breast cancer survivorship. This scoping review examines the evidence on the influence of psychosocial determinants on QoL in breast cancer survivors.

Methods Scoping review methodology was used to: (1) identify the research question(s); (2) identify relevant studies; (3) undertake study selection; (4) extract data; (5) collate, summarise and report the results.

Results A total of 34 studies met the inclusion criteria. The majority of studies were conducted in the US $(n=23,68 \%)$ and were mainly cross-sectional $(n=27,79 \%)$. Sixteen psychosocial determinants of QoL were identified. Social support $(n=16,47 \%)$, depression $(n=7,21 \%)$ and future appraisal and perspective $(n=7,21 \%)$ were the most frequently investigated determinants. Eighteen different QoL measures were used. A range of different measurement tools were also used per psychosocial determinant (weighted average $=6$ ). The 16 studies that measured the influence of social support on QoL employed 11 different measures of social support and 10 different measures of QoL. In general, across all 34 studies, a higher level of a positive influence and a lower level of a negative influence of a psychosocial determinant was associated with a better QoL e.g. higher social support and lower levels of depression were associated with a higher/better QoL. For some determinants such as spirituality and coping skills the influence on QoL varied, but these determinants were less commonly investigated.

Conclusion Consensus around measures of QoL and psychological determinants would be valuable and would enable research to determine the influence of psychosocial determinants on QoL adequately. Research in other healthcare settings beyond the US is required, in order to understand the influence of organisation and follow-up clinical and supportive care on psychosocial determinants and QoL and to improve the quality of care in breast cancer survivors.

\section{P09 DEVELOPMENT OF A COMMUNITY-BASED INTERVENTION TO INCREASE UPTAKE OF HOME BOWEL CANCER SCREENING IN SOUTH ASIANS: A MIXED METHODS STUDY}

${ }^{1} \mathrm{~L}$ Smith ${ }^{*}{ }^{2} \mathrm{~S}$ Howcutt, ${ }^{3} \mathrm{P}$ Saini, ${ }^{2,4} \mathrm{~J}$ Brett, ${ }^{2,4} \mathrm{C}$ Henshall, ${ }^{2,4} \mathrm{E}$ Watson. ${ }^{1} \mathrm{C}$ CAHR, Faculty of Health Sciences, University of Hull, Hull, UK; ${ }^{2}$ Faculty of Health and Life Sciences, Oxford Brookes University, Oxford, UK; ${ }^{3}$ School of Psychology Faculty of Health, Liverpool John Moores University, Liverpool, UK; ${ }^{4}$ OXINMAHR, Faculty of Health and Life Sciences, Oxford Brookes University, Oxford, UK

\subsection{6/jech-2020-SSMabstracts. 105}

Background Bowel cancer is common and accounts for $10 \%$ of all cancer mortality-second only to lung cancer deaths. If detected early through screening tests, mortality is significantly reduced. The NHS Bowel Cancer Screening Programme (BCSP) invites adults aged 60-74 years to carry out a home screening test biennially. The national target for test completion is 60\%; completion is substantially lower ( 30\%) amongst South Asian populations.

Universal approaches to increase screening uptake, e.g. text reminders, are effective, but may widen health inequalities, as they tend to benefit individuals with greater agency. Strategies tailored to ethnic minority groups show promise but are poorly specified and evidence of effectiveness is lacking. Limited evidence suggests that barriers to bowel cancer screening for South Asians are complex.

The aim was to develop a community-based intervention to increase completion of the home bowel screening test in South Asians. 